\title{
On the Performance of Multiuser Scheduling with Post-Examining Under Non-Identical Fading
}

\author{
Fakhreddine Gaaloul*, Redha M. Radaydeh*, Mohamed-Slim Alouini* and Hong-Chuan Yang\# \\ *Division of Physical Sciences and Engineering \\ King Abdullah University of Science and Technology (KAUST) \\ Thuwal, Makkah Province, Kingdom of Saudi Arabia \\ Email: \{gaaloul.fakhreddine, redha.radaydeh, slim.alouini\} @kaust.edu.sa \\ \# Department of Electrical and Computer Engineering \\ University of Victoria \\ Victoria BC, V8W 3P6, Canada \\ Email: hyang@ece.uvic.ca
}

\begin{abstract}
We investigate the performance of a multiuser downlink access scheme based on a post-selection switch and examine algorithm. The studied scheme sequentially switches over the users that experience independent and non-identically distributed fading conditions, and selects a single user with an acceptable channel quality as compared to a pre-selected signal-to-noise ratio (SNR) threshold. If no one of the users can satisfy the target channel quality, the base station (BS) takes the advantage of the knowledge of all users channels and serves the user with the best channel quality among all users. This scheme reduces considerably the feedback load but offers a lower average spectral efficiency (ASE) as compared to that of the full feedback system with instantaneous best user selection. On the other hand, it improves the system performances, such as outage probability and average bit error rate (BER), as compared to a system that is based on a standard switching scheme without post-selection. Numerical results for the ASE average BER, and average feed back load, are presented for the cases of outdated and non-outdated rate-adaptive modulation scheme operating over independent and non-identically distributed users.
\end{abstract}

\section{INTRODUCTION}

By exploiting the users channel fluctuation, multiuser diversity has been proposed to provide a service to the user with the best channel in a multiuser environment [1]. This technique improves the throughput of wireless systems at the expense of large overhead. Particularly, feedback from all active mobile stations (MSs) is needed in order to keep the base station (BS) updated with the channel state of each mobile terminal. Several opportunistic scheduling schemes has been proposed and analyzed for independent and identically distributed users [2]. For instance, with the use of the best selection algorithm, the BS probes sequentially all the users and select the user with the best channel. This scheme has a high average spectral efficiency (ASE) and provides a high data rate for the selected user, but it requires full feedback information from all users to operate adequately. To reduce the feedback load from different MSs, switching schemes have been proposed [3]-[5], in which the BS, which operates in a time division multiplexing (TDM) mode, probes sequentially the users channels and selects the first user whose predicted signal-to-noise ratio (SNR) is above a pre-selected design parameter threshold.

This paper adds enhancements on the aforementioned contributions, and investigates a multiuser scheduling scheme that is based on post-selection switch and examine algorithm. The case study under consideration assumes a more general scenario of that adopted in [5], for which the users channels are assumed to be independent but non-identically distributed (i.n.d.). The post-selection switch and examine algorithm adopted herein operates as follow: the BS starts sequentially probing the users by requesting each user SNR until a user with an instantaneous SNR above the threshold is identified. When the instantaneous SNRs of all tested users are found below the threshold, the BS selects the user with the best channel quality for subsequent packet transmission. Moreover, this scheduler can be much more efficient when implementing an adaptive modulation scheme. The adaptation to channel conditions is proposed based on the active users instantaneous SNRs to choose from different quadrature-amplitude constellations over discrete-time intervals, while reducing the feedback load.

For the scheduling scheme under investigation, three performance metrics are studied, which are the ASE, the average feedback load (AFL), and the average bit error rate (BER) for the adaptive system. These performance metrics are shown for a generic rate-adaptive modulation scheme, and their corresponding numerical results are presented for adaptive rectangular signaling with finite constellations. In addition, more realistic results are provided in this paper for the BER when the channel estimates are outdated or imperfect due to the effect of scheduling delay. To obtain such results, a new approach based on the conditional moment generating function (MGF) is proposed.

The remainder of the paper is organized as follows. Section II outlines the system model, and presents the statistics of the resulting SNR of the selected user for non-adaptive algorithm with post-examining selection under i.n.d. channel conditions. Section III provides analytical results for the ASE, average BER, and AFL for the SNR-based adaptive system. Section IV quantifies the effect of imperfect channel state 
informations (CSIs) on the statistics of the resulting SNR as well as the average BER, and section $\mathrm{V}$ shows selected numerical and simulation results.

\section{System Model and Statistics of Resulting SNR}

This section outlines the system model and presents the general formulations for the resulting SNR of the selected user per the scheduling algorithm under consideration. The results are then used to study the case when the examined users undergo i.n.d. Rayleigh fading.

\section{A. System Model}

It is assumed that a total of $\mathrm{L}$ users exist in the coverage area of interest. The users can receive downlink information from a known BS, which is equipped with a single antenna and can use a single channel to serve one of the active users at a time. The mobile stations are assumed to be space-limited, and each of which has only one antenna to receive and transmit in time division duplex (TDD) fashion, thereby the channel reciprocity can be exploited to extract the CSI of each examined user.

During each guard period, the BS listens to the requests from active users sequentially and arbitrarily, and aims to serve a user whose channel quality is good enough to support reliable reception of the projected signaling constellation. Knowing that the projected performance can be mapped into a specific threshold on the resulting instantaneous SNR, the BS starts probing the users sequentially, where the estimated instantaneous SNR of the firstly probed user is compared against the SNR threshold. If it is found that the first user instantaneous SNR is above the threshold, the first user is allocated the available packet interval, and no further processing is required. Otherwise, the BS probes another user and examines its estimated instantaneous SNR against the threshold. The BS continues examining the instantaneous SNRs of the probed users until either one user having instantaneous SNR above the threshold is found or all active users have been examined. In the latter case, the BS selects the user having the best SNR, and allocate the constellation size so that this user can decode reliably with the specified target error rate.

\section{B. Statistics of Resulting SNR}

Consider a system with $L$ users, where the $l$ th user has an instantaneous SNR of $\gamma_{l}$, where $l=1,2, . ., L$. Let $\Gamma$ be the resulting (i.e. output) SNR. When the post-selection switch and examine algorithm is used at the serving BS, the cumulative distribution function (CDF) of the output SNR for the general case of i.n.d. channel gains of active users can be written as

$$
\begin{aligned}
F_{\Gamma}(x)= & \prod_{l=1}^{L} F_{\gamma_{l}}(x)\left(U(x)-U\left(x-\gamma_{T}\right)\right) \\
& +\left[\sum_{l=1}^{L} \prod_{i=1}^{l-1} F_{\gamma_{i}}\left(\gamma_{T}\right)\left[F_{\gamma_{l}}(x)-F_{\gamma_{l}}\left(\gamma_{T}\right)\right]\right. \\
& \left.+\prod_{l=1}^{L} F_{\gamma_{l}}\left(\gamma_{T}\right)\right] U\left(x-\gamma_{T}\right)
\end{aligned}
$$

where $F_{\gamma_{l}}(x)$, for $l=1,2, . ., L$, is the CDF of the instantaneous SNR associated with the $l$ th user, $\gamma_{T}$ is a pre-selected threshold, and $U($.$) is the unit step function. The probability$ density function (PDF) of the output SNR can be obtained from (1), and the result is

$$
\begin{aligned}
f_{\Gamma}(x)= & \sum_{l=1}^{L}\left(f_{\gamma_{l}}(x) \prod_{\substack{i=1 \\
i \neq l}}^{L} F_{\gamma_{i}}(x)\left(U(x)-U\left(x-\gamma_{T}\right)\right)\right. \\
& \left.+\left(\prod_{i=1}^{l-1} F_{\gamma_{i}}\left(\gamma_{T}\right)\right) f_{\gamma_{l}}(x) U\left(x-\gamma_{T}\right)\right),
\end{aligned}
$$

where $f_{\gamma_{l}}(x) \triangleq d F_{\gamma_{l}}(x) / d x$, for $l=1,2, . ., L$, is the PDF of the instantaneous SNR associated with the lth user.

Using the statistics the output SNR presented above, the MGF of $\Gamma$ can be obtained as

$$
M_{\Gamma}(s)=1-s \int_{0}^{\infty} e^{-s x} \bar{F}_{\Gamma}(x) d x=\int_{0}^{\infty} e^{-s x} f_{\Gamma}(x) d x
$$

where $\bar{F}_{\Gamma}(x)=1-F_{\Gamma}(x)$ is the complementary cumulative distribution function (CCDF) of $\Gamma$.

\section{Application to Rayleigh Fading Environment}

For the case when the channel gains of individual users undergo Rayleigh fading, the PDF and the CDF of the users instantaneous SNRs are given by $f_{\gamma_{l}}(x)=\frac{1}{\bar{\gamma}_{l}} e^{-\frac{x}{\bar{\gamma}_{l}}}, x \geq 0$, and $F_{\gamma_{l}}(x)=1-e^{-\frac{x}{\bar{\gamma}_{l}}}, x \geq 0$, where $\bar{\gamma}_{l}$, for $l=1, . ., L$, is the average SNR of the $l$ th channel. The results of $f_{\gamma_{l}}(x)$ and $F_{\gamma_{l}}(x)$ given above can be used in (2) and (1) to find the PDF and the CDF of the output SNR when the channel gains of the $L$ users undergo i.n.d. Rayleigh fading, respectively. The MGF of $\Gamma$ can be written as

$$
M_{\Gamma}(s)=M_{\Gamma}(s, 0)
$$

where $M_{\Gamma}(s, \gamma)$ represents the incomplete MGF and given by $M_{\Gamma}(s, \gamma)=\int_{\gamma}^{+\infty} e^{-s x} f_{\Gamma}(x) d x$. Note that the product $\prod_{i=1, i \neq l}^{L} F_{\gamma_{i}}(x)$ in (2) can be expressed as

$$
\prod_{\substack{i=1 \\ i \neq l}}^{L} F_{\gamma_{i}}(x)=1+\sum_{i=1}^{R}(-1)^{\xi} e^{-A_{i, l} x}+(-1)^{L-1} e^{-\sum_{\substack{i=1 \\ i \neq l}}^{L} \frac{1}{\bar{\gamma}_{i}} x}
$$

where $A_{i, l}$ is the $i$ th element of a set $A$ that contains the sum of all the $n$-tuples for $n=1,2, \ldots, L-2$ of the set $S$ defined by $S=\left\{\frac{1}{\bar{\gamma}_{1}}, \frac{1}{\bar{\gamma}_{2}}, \ldots, \frac{1}{\bar{\gamma}_{L}}\right\} / \frac{1}{\bar{\gamma}_{l}}, \xi$ is the number of the summed element in $A_{i, l}$ and $R$ is the number of element of the set $A$ given by $R=\sum_{l=1}^{L-2}\left(\begin{array}{c}L-1 \\ l\end{array}\right)$. Using the representation in (5), $M_{\Gamma}(s, \gamma)$ can be easily calculated as

$$
M_{\Gamma}(s, \gamma)=\sum_{l=1}^{L} M_{\Gamma_{l}}(s, \gamma),
$$


where $M_{\Gamma_{l}}(s, \gamma)$ is the incomplete MGF of $\Gamma$ when the $l$ th user is selected, and it can be expressed as

$$
\begin{aligned}
& M_{\Gamma_{l}}(s, \gamma)=A_{l}(\gamma) U\left(\gamma-\gamma_{T}\right)+\left[A_{l}\left(\gamma_{T}\right)+\frac{1}{\bar{\gamma}_{l}}\left(\left(s+\frac{1}{\bar{\gamma}_{l}}\right)^{-1}\right.\right. \\
& \times \Phi\left(\gamma, \gamma_{T},\left(s+\frac{1}{\bar{\gamma}_{l}}\right)\right)+\sum_{r=1}^{R}(-1)^{\xi}\left(A_{r}+\frac{1}{\bar{\gamma}_{l}}+s\right)^{-1} \\
& \times \Phi\left(\gamma, \gamma_{T},\left(A_{r}+s+\frac{1}{\bar{\gamma}_{l}}\right)\right)+(-1)^{L-1}\left(\sum_{j=1}^{L} \frac{1}{\bar{\gamma}_{j}}+s\right)^{-1} \\
& \left.\left.\times \Phi\left(\gamma, \gamma_{T},\left(\sum_{j=1}^{L} \frac{1}{\bar{\gamma}_{j}}+s\right)\right)\right)\right]\left(U(\gamma)-U\left(\gamma-\gamma_{T}\right)\right)
\end{aligned}
$$

where $A_{l}(\gamma)$ and $\Phi(x, y, z)$ are given by $A_{l}(\gamma)=\left(\prod_{i=1}^{l-1}(1-\right.$ $\left.\left.e^{-\frac{\gamma_{T}}{\gamma_{i}}}\right)\right) \frac{e^{-\left(s+\frac{1}{\gamma_{\gamma}}\right) \gamma}}{1+s \bar{\gamma}_{l}}$ and $\Phi(x, y, z)=e^{-x z}-e^{-y z}$.

\section{Adaptive Scheduling With Post-Examining SELECTION FOR I.N.D. USERS}

This section considers the use of the scheduling algorithm with post-examining selection described in the previous section in combination with discrete-time variable-rate coded quadrature-amplitude modulation. Specifically, the range of the resulting instantaneous SNR is divided into $N+1$ regions, which are referred to as $\left\{R_{n}\right\}_{n=1}^{N}$, for which the fixed set of adaptation thresholds $\left\{\gamma_{1}^{*}, \gamma_{2}^{*}, \cdots, \gamma_{N}^{*}, \gamma_{N+1}^{*}\right\}$ are used to define the boundary points, where $\gamma_{N+1}^{*}=+\infty$. In this case, the constellation of size $M_{n} \triangleq 2^{R_{n}}$ is chosen when it it is found that $\Gamma \in R_{n}=\left[\gamma_{n}^{*}, \gamma_{n+1}^{*}\right)$ such that a target BER is satisfied. The following parts discusses the analytical formulations of performance and processing complexity measures, respectively.

\section{A. Performance Measures}

The expression for the ASE can be obtained as ASE = $\sum_{n=1}^{N} R_{n} P_{n}$, where $P_{n}=F_{\Gamma}\left(\gamma_{n+1}\right)-F_{\Gamma}\left(\gamma_{n}\right)$ refers to the probability that $\Gamma \in R_{n}$, and $F_{\Gamma}($.$) is given in a general form$ in (1). The average BER can be obtained as

$$
\overline{\mathrm{BER}}=\frac{\sum_{n=1}^{N} R_{n} \overline{\mathrm{BER}}_{n}}{\mathrm{ASE}},
$$

where $\overline{\mathrm{BER}}_{n}=\mathbb{E}\left\{\mathrm{BER}_{n} \mid \Gamma \in R_{n}\right\}$, and $\mathrm{BER}_{n}$ for the signaling constellation under consideration can be written as $\mathrm{BER}_{n}=a_{n} e^{-b_{n} \gamma / M_{n}}$ [8], in which where $a_{n}, b_{n}$ and $M_{n}$ are region-dependent constants that are related to the constellation size chosen for the $n$th region. Then, it can be written that

$$
\begin{aligned}
\overline{\mathrm{BER}}_{n} & =\int_{\gamma_{n}}^{\gamma_{n+1}} a_{n} e^{-b_{n} \gamma / M_{n}} f_{\Gamma}(\gamma) d \gamma \\
& =a_{n}\left[M_{\Gamma}\left(\frac{b_{n}}{M_{n}}, \gamma_{n}\right)-M_{\Gamma}\left(\frac{b_{n}}{M_{n}}, \gamma_{n+1}\right)\right],
\end{aligned}
$$

where $M_{\Gamma}(s, \gamma)$ is given in (7).

\section{B. Processing Load Measure}

Define $N_{e}$ as a discrete-valued random variable that takes values from the set $\{1,2, \ldots, L\}$, and it is used to refer to the number of users need to be examined per each packet transmission. For a given value of the SNR threshold $\gamma_{n}^{*}$, it can be shown, for the case i.n.d. users, that $\operatorname{Pr}\left\{N_{e}=\right.$ $K\}=\prod_{k=1}^{K-1} F_{\gamma_{k}}\left(\gamma_{n}^{*}\right)\left[1-F_{\gamma_{K}}\left(\gamma_{n}^{*}\right)\right]$, for $K=1, . ., L-1$, and $\operatorname{Pr}\left\{N_{e}=L\right\}=\prod_{k=1}^{L-1} F_{\gamma_{k}}\left(\gamma_{n}^{*}\right)$. The expression for the AFL can be then obtained as

$$
\bar{N}_{e}=\sum_{K=1}^{L-1} K \prod_{k=1}^{K-1} F_{\gamma_{k}}\left(\gamma_{n}^{*}\right)\left[1-F_{\gamma_{K}}\left(\gamma_{n}^{*}\right)\right]+L \prod_{k=1}^{L-1} F_{\gamma_{k}}\left(\gamma_{n}^{*}\right),
$$

which is generally applicable for any channel fading models.

\section{EFfect of Outdated Channel estimates}

In a practical system, and because of real world implementation constraints, channel estimates are usually outdated or imperfect. In this case, the results obtained in the preceding sections under the assumption of on-outdated SNRs of individual users are no longer valid. In fact, outdated channel estimation occurs when the transmitter does not have a perfect channel estimate at time $t+\tau$, which is the time to transmit after selecting the adequate user, but have only a channel estimate at time $t$, where $\tau$ represents the time delay which is exactly equal to the scheduling duration. Consequently, when using adaptive modulation techniques at the transmitter, the link adaptation at time $t+\tau$ will be based on the estimated channel at time $t$, and thus the delay will affect the choice of the constellation size, leading to performance degradation.

This section obtains the statistics of the output SNR when channel estimation is outdated or imperfect. Assuming that the channels of the $L$ users are i.n.d., and $\Gamma^{(\tau)}$ be the actual signal SNR when the transmission takes place. Due to imperfect channel estimation caused by feedback delay, $\Gamma^{(\tau)}$ may or may not be the same as $\Gamma$, as discussed above.

\section{A. PDF of Outdated Output SNR}

Noting that $\Gamma$ and $\Gamma^{(\tau)}$ correspond to the SNR of the selected user, the PDF of $\Gamma_{l}^{(\tau)}$, for $l=1, . ., L$, can be written as

$$
f_{\Gamma_{l}^{(\tau)}}(x)=\int_{0}^{+\infty} f_{\Gamma_{l}}(u) \frac{f_{\Gamma_{l}, \Gamma_{l}^{(\tau)}}(u, x)}{f_{\gamma_{l}}(x)} d u
$$

where $f_{\Gamma_{l}}($.$) is the PDF of the \Gamma$ when the $l$ th user is chosen, which can be read from (2) as

$$
\begin{aligned}
f_{\Gamma_{l}}(x) & =f_{\gamma_{l}}(x) \prod_{i=1, i \neq l}^{L} F_{\gamma_{i}}(x)\left(U(x)-U\left(x-\gamma_{T}\right)\right) \\
& +\left(\prod_{i=1}^{l-1} F_{\gamma_{i}}\left(\gamma_{T}\right)\right) f_{\gamma_{l}}(x) U\left(x-\gamma_{T}\right) .
\end{aligned}
$$


The PDF of $\Gamma^{(\tau)}=\sum_{l=1}^{L} \Gamma_{l}^{(\tau)}$ can now be written as

$$
\begin{aligned}
f_{\Gamma^{(\tau)}}(x) & =\sum_{l=1}^{L}\left[\int_{0}^{\gamma_{T}} \prod_{\substack{i=1 \\
i \neq j}}^{L} F_{\gamma_{i}}(u) f_{\Gamma_{l}, \Gamma_{l}^{(\tau)}}(u, x) d u\right. \\
& \left.+\left(\prod_{i=1}^{l-1} F_{\gamma_{i}}\left(\gamma_{T}\right)\right) \int_{\gamma_{T}}^{+\infty} f_{\Gamma_{l}, \Gamma_{l}^{(\tau)}}(u, x) d u\right]
\end{aligned}
$$

For the case of Rayleigh fading, the joint PDF, $f_{\Gamma_{l}, \Gamma_{l}^{(\tau)}}(u, x)$, is given by [12, eq.(126)]

$$
f_{\Gamma_{l}, \Gamma_{l}^{(\tau)}}\left(x_{1}, x_{2}\right)=\frac{e^{-\frac{\left(x_{1}+x_{2}\right)}{\left(1-\rho_{l}\right) \bar{\gamma}_{l}}}}{\left(1-\rho_{l}\right) \bar{\gamma}_{l}^{2}} I_{0}\left(2 \sqrt{\frac{\rho x_{1} x_{2}}{\left(1-\rho_{l}\right)^{2} \bar{\gamma}_{l}^{2}}}\right),
$$

where $I_{0}($.$) is the zero-order modified Bessel function of$ the first kind, $0 \leq \rho_{l} \leq 1$, for $l=1, \ldots, L$, denote the power correlation coefficients between $\Gamma_{l}$ and $\Gamma_{l}^{(\tau)}$. The value of $\rho_{l}$ may be expressed as a function of the delay $\tau$ as $\rho=J_{0}^{2}\left(2 \pi f_{D} \tau\right)$, where $J_{0}($.$) is the zero-order Bessel function$ of the first kind and $f_{D}=v / \lambda$ is the maximum Doppler frequency shift, where $v$ is the speed of the corresponding user and $\lambda$ is the wavelength of the operating frequency. The PDF of the outdated output SNR in (13) can be expressed as

$$
f_{\Gamma^{(\tau)}}(x)=\sum_{l=1}^{L}\left[f_{\Gamma_{l}^{(\tau)}}^{(1)}(x)+\left(\prod_{i=1}^{l-1} F_{\gamma_{i}}\left(\gamma_{T}\right)\right) f_{\Gamma_{l}^{(\tau)}}^{(2)}(x)\right]
$$

where $f_{\Gamma_{l}^{(\tau)}}^{(1)}(x)$ and $f_{\Gamma_{l}^{(\tau)}}^{(2)}(x)$ are given by

$$
\begin{aligned}
& f_{\Gamma_{j}^{(\tau)}}^{(1)}(x)=\int_{0}^{\gamma_{T}} \prod_{\substack{i=1 \\
i \neq j}}^{L} F_{\gamma_{i}}(u) f_{\Gamma_{j}, \Gamma_{j}^{(\tau)}}(u, x) d u \\
& =\widetilde{F}_{j}(0, x)+\sum_{l=1}^{R}(-1)^{\xi} \widetilde{F}_{j}\left(A_{l, j}, x\right) \\
& +(-1)^{L-1} \widetilde{F}_{j}\left(\sum_{l=1, l \neq j}^{L} \frac{1}{\bar{\gamma}_{l}}, x\right) \\
& f_{\Gamma_{j}^{(\tau)}}^{(2)}(x)=\frac{1}{\bar{\gamma}_{j}} e^{-\frac{x}{\bar{\gamma}_{j}}}-\widetilde{F}_{j}(0, x)
\end{aligned}
$$

where $A_{l, j}$ are the elements of the set $A_{j}$ containing all the $n$-tuples when $n=1, . ., L-1$ of the element of the set $S_{j}=$ $S /\left\{\frac{1}{\bar{\gamma}_{j}}\right\}$ and $S=\bar{\gamma}_{j}, j=1, . ., L$. In (16), $\widetilde{F}_{j}(.,$.$) is given by$

$$
\begin{aligned}
& \widetilde{F}_{j}(r, x)=\frac{1}{\bar{\gamma}_{j}\left(r\left(1-\rho_{j}\right) \bar{\gamma}_{j}+1\right)} e^{-\left(\frac{1+r \bar{\gamma}_{j}}{\bar{\gamma}_{j}\left(r\left(1-\rho_{j}\right) \bar{\gamma}_{j}+1\right)}\right) x} \\
& \times\left(1-Q\left(\sqrt{\frac{2 \rho_{j} x}{\left(1-\rho_{j}\right) \bar{\gamma}_{j}\left(r\left(1-\rho_{j}\right) \bar{\gamma}_{j}+1\right)}}\right.\right. \\
& \left.\left.\sqrt{2 \gamma_{T}\left(r+\frac{1}{\left(1-\rho_{j}\right) \bar{\gamma}_{j}}\right)}\right)\right)
\end{aligned}
$$

where $Q(.,$.$) is the Q-Marcum function [13].$

\section{B. MGF of the Outdated Output SNR}

The MGF of $\Gamma^{(\tau)}$ can be obtained with the help of (3) as

$$
\begin{aligned}
& M_{\Gamma^{(\tau)}}(s)=\int_{0}^{+\infty} e^{-s x} f_{\Gamma^{(\tau)}}(x) d x \\
& =\sum_{j=1}^{L}\left[M_{\Gamma_{j}^{(\tau)}}^{(1)}(s)+\left(\prod_{i=1}^{j-1} F_{\gamma_{i}}\left(\gamma_{T}\right)\right) M_{\Gamma_{j}^{(\tau)}}^{(2)}(s)\right],
\end{aligned}
$$

where $M_{\Gamma_{j}^{(\tau)}}^{(1)}(s)$ and $M_{\Gamma_{j}^{(\tau)}}^{(2)}(s)$ are given by

$$
\begin{aligned}
M_{\Gamma_{j}^{(\tau)}}^{(1)}(s) & =\widetilde{M}_{j}(0, s)+\sum_{l=1}^{R}(-1)^{\xi} \widetilde{M}_{j}\left(A_{l, j}, s\right) \\
& +(-1)^{L-1} \widetilde{M}_{j}\left(\sum_{l=1, l \neq j}^{L} \frac{1}{\bar{\gamma}_{l}}, s\right) \\
M_{\Gamma_{j}^{(\tau)}}^{(2)}(s) & =\frac{1}{\bar{\gamma}_{j}}\left(s+\frac{1}{\bar{\gamma}_{j}}\right)^{-1}-\widetilde{M}_{j}(0, s),
\end{aligned}
$$

where $\widetilde{M}_{j}(.,$.$) is given by$

$$
\begin{aligned}
\widetilde{M}_{j}(r, s) & =\frac{1}{\left(1-\rho_{j}\right) \bar{\gamma}_{j}^{2}}\left(r+\frac{1}{\left(1-\rho_{j}\right) \bar{\gamma}_{j}}\right)^{-1} \\
& \times a^{-1}\left[1-\Gamma\left(1-\frac{c a}{2 a+b}\right)\right]
\end{aligned}
$$

in which $a, b$ and $c$ are given by $a=\frac{1}{\left(1-\rho_{j}\right) \bar{\gamma}_{j}}-$ $\frac{\rho_{j}}{\left(1-\rho_{j}\right) \bar{\gamma}_{j}\left(r\left(1-\rho_{j}\right)+1\right)}+s, b=\frac{2 \rho_{j}}{\left(1-\rho_{j}\right) \bar{\gamma}_{j}\left(r\left(1-\rho_{j}\right) \bar{\gamma}_{j}+1\right)}$, and $c=$ $2 \gamma_{T}\left(r+\frac{1}{\left(1-\rho_{j}\right) \bar{\gamma}_{j}}\right)$.

\section{Average BER Under the Effect of Outdated Output SNR}

This part studies the effect of outdated estimates of the output SNR on the average BER performance of the rateadaptive transmission. In this case, it can be written that

$$
\overline{\mathrm{BER}}=\frac{\sum_{n=1}^{N} R_{n} \overline{\mathrm{BER}}_{n}^{\prime}}{\mathrm{ASE}}
$$

where $\overline{\mathrm{BER}}_{n}^{\prime}=\sum_{l=1}^{L} \overline{\mathrm{BER}}_{n, l}^{\prime}$ is the average BER experienced when modulation index $n$ is applied, which is the only component affected by the delay. The term $\overline{\mathrm{BER}}_{n, l}^{\prime}$ can be expressed as

$$
\begin{aligned}
\overline{\operatorname{BER}}_{n, l}^{\prime} & =\int_{\gamma_{n}}^{\gamma_{n+1}} \int_{0}^{\infty} \operatorname{BER}\left(a_{n}, b_{n}, M_{n}, \gamma_{\tau}\right) \\
& \times f_{\Gamma_{l}^{(\tau)} / \Gamma_{l}}\left(\gamma_{\tau} / \gamma\right) d \gamma_{\tau} f_{\Gamma_{l}}(\gamma) d \gamma \\
& =\int_{\gamma_{n}}^{\gamma_{n+1}} \operatorname{BER}_{\Gamma_{l}^{(\tau)} / \Gamma_{l}}\left(a_{n}, b_{n}, M_{n}, \gamma\right) f_{\Gamma_{l}}(\gamma) d \gamma
\end{aligned}
$$

where $f_{\Gamma_{l}}(\gamma)$ is the PDF of $\Gamma$, which is the output SNR at the $\mathrm{BS}$, when the $l$ th user is selected and is given by (12). The term $f_{\Gamma_{l}^{(\tau)} / \Gamma_{l}}\left(\gamma_{\tau} / \gamma\right)$ represents the conditioned PDF which can 
be easily calculated, using the joint PDF $f_{\Gamma_{l}^{(\tau)}, \Gamma_{l}}\left(\gamma_{\tau}, \gamma\right)$ given by (14), as follows:

$$
\begin{aligned}
& f_{\Gamma_{l}^{(\tau)} / \Gamma_{l}}\left(\gamma_{\tau} / \gamma\right)=\frac{f_{\Gamma_{l}^{(\tau)}, \Gamma_{l}}\left(\gamma_{\tau}, \gamma\right)}{f_{\gamma_{l}}(\gamma)} \\
& =\frac{1}{\left(1-\rho_{l}\right) \bar{\gamma}_{l}} e^{-\frac{\rho_{l} \gamma+\gamma_{\tau}}{\left(1-\rho_{l}\right) \bar{\gamma}_{l}}} I_{0}\left(2 \frac{\sqrt{\rho_{l} \gamma \gamma_{\tau}}}{\left(1-\rho_{l}\right) \bar{\gamma}_{l}}\right) .
\end{aligned}
$$

Given that $\operatorname{BER}\left(a_{n}, b_{n}, M_{n}, \gamma\right)=a_{n} e^{-\frac{b_{n}}{M_{n}} \gamma}$ as defined previously, the term $\operatorname{BER}_{\Gamma_{l}^{(\tau)} / \Gamma_{l}}\left(a_{n}, b_{n}, M_{n}, \gamma\right)$ can be represented in a more general form using the conditional MGF as $\operatorname{BER}_{\Gamma_{l}^{(\tau)} / \Gamma_{l}}\left(a_{n}, b_{n}, M_{n}, \gamma\right)=a_{n} M_{\Gamma_{l}^{(\tau)} / \Gamma_{l}}\left(\frac{b_{n}}{M_{n}}, \gamma\right)$. The conditional MGF, $M_{\Gamma_{l}^{(\tau)} / \Gamma_{l}}(s, \gamma)$, can be easily calculated using the condition PDF in (23) and with the help of [11], and the result is

$$
\begin{aligned}
M_{\Gamma_{l}^{(\tau)} / \Gamma_{l}}(s, \gamma) & =\int_{0}^{\infty} e^{-s \gamma_{\tau}} f_{\Gamma_{l}^{(\tau)} / \Gamma_{l}}\left(\gamma_{\tau} / \gamma\right) d \gamma_{\tau} \\
& =\frac{1}{1+s \bar{\gamma}_{l}\left(1-\rho_{l}\right)} e^{-\frac{\rho_{l} s \gamma}{1+s / \bar{\gamma}_{l}\left(1-\rho_{l}\right)}} .
\end{aligned}
$$

The final result of the average BER can be represented as a function of the incomplete MGF defined in (7). That gives

$$
\begin{aligned}
& \overline{\mathrm{BER}}=\frac{1}{\mathrm{ASE}} \sum_{n=1}^{N} R_{n} \sum_{l=1}^{L} \alpha_{n, l} \\
& \times\left[M_{\Gamma_{l}}\left(\beta_{n, l}, \gamma_{n}\right)-M_{\Gamma_{l}}\left(\beta_{n, l}, \gamma_{n+1}\right)\right],
\end{aligned}
$$

where $\alpha_{n, l}$ and $\beta_{n, l}$, for $l=1, . ., L$ and $n=1, . ., N$, are constants, which depend on $\left\{\rho_{l}\right\},\left\{\bar{\gamma}_{l}\right\}$, and the adaptive signalling constants (i.e. $\left\{a_{n}\right\},\left\{b_{n}\right\}$, and $\left\{M_{n}\right\}$ ). These parameters are given by $\alpha_{n, l}=\frac{a_{n} M_{n}}{M_{n}+b_{n} \bar{\gamma}_{l}\left(1-\rho_{l}\right)}$ and $\beta_{n, l}=\frac{\rho_{l} b_{n}}{M_{n}+b_{n} \bar{\gamma}_{l}\left(1-\rho_{l}\right)}$.

\section{Numerical RESUlts AND Discussions}

This section presents some numerical results of the performance metrics discussed in the preceding sections. In generating the numerical results, it is assumed that the users are uniformly distributed in a cell of radius $R$ centered around a BS. Each user has different average SNR, $\bar{\gamma}_{l}$ for $l=1, . ., L$, which is assumed to be log-normal distributed with shadowing standard deviation of $\theta=3 \mathrm{~dB}$ in the curves shown. For the case under consideration, the PDF of the average SNR $\bar{\gamma}_{l}$, for $l=1, . ., L$, can be given by [10]

$$
f_{\bar{\gamma}_{l}}(\bar{\gamma})=\frac{2}{c} e^{\frac{2 \theta^{2}-2 c\left(\bar{\gamma}-\bar{\gamma}_{R}\right)}{c^{2}}} Q\left(\frac{2 \theta^{2}-c\left(\bar{\gamma}-\bar{\gamma}_{R}\right)}{c \theta}\right),
$$

where $\bar{\gamma}_{R}$ is the average SNR at distance $R, \alpha$ is the path loss exponent ( $\alpha=3.5$ in the numerical results), $c=10 \alpha \log (e)$ and $Q($.$) is the one-dimensional Gaussian Q-function.$

For the ASE and average BER performance results, an 8 region (i.e. $N=8$ ) adaptive discrete rate rectangular signalling scheme [8] is assumed with a target $\mathrm{BER}_{0}=$ $10^{-3}$ and $\left\{R_{n}\right\}_{n=1}^{8}=[1,2,3,4,5,6,7,8]$. For this considered scheme, the constant $a_{n}, b_{n}$ and $M_{n}$ defined previously are given by: $a_{n}=0.2, b_{n}=3 / 2$ and $M_{n}=$ $M_{n}^{\prime}-1$, where $M_{n}^{\prime}=[2,4,8,16,32,64,128,256]$ represents the M-QAM constellation size for each region.
The regions threshold $\left[\gamma_{1}, \gamma_{2}, . ., \gamma_{N}\right]$ are calculated using the target $\mathrm{BER}_{0}$, according to [8], and are given by $[6.78,10.25,13.93,17.24,20.39,23.47,26.51,29.54] \mathrm{dB}$.

In Fig. 1, the ASE is plotted as a function of the average SNR at a distance $R$ from the base station, $\bar{\gamma}_{R}$. Also, the ASE is depicted for different values of switching threshold $\gamma_{T}$ for a target BER of $10^{-3}$ where we assume that the number of users in the cell $L=5$. It can be seen from the figure that as $\bar{\gamma}_{R}$ increases the ASE increases and this is because increasing $\bar{\gamma}_{R}$ is equivalent to decreasing the radius of the cell, thus the different users are closer to the BS and their channels are better which explain the increase of the ASE. Fig. 1 shows also that as the switching threshold increases the ASE is improved and approaches the ASE of the best selection algorithm which gives the best ASE (the ASE of the best selection algorithm can be obtained by setting $\gamma_{T} \rightarrow+\infty$ ). This can be explained by the fact that when increasing $\gamma_{T}$ the BS selects a user with a better channel which will transmit with a better rate and as such the ASE is improved.

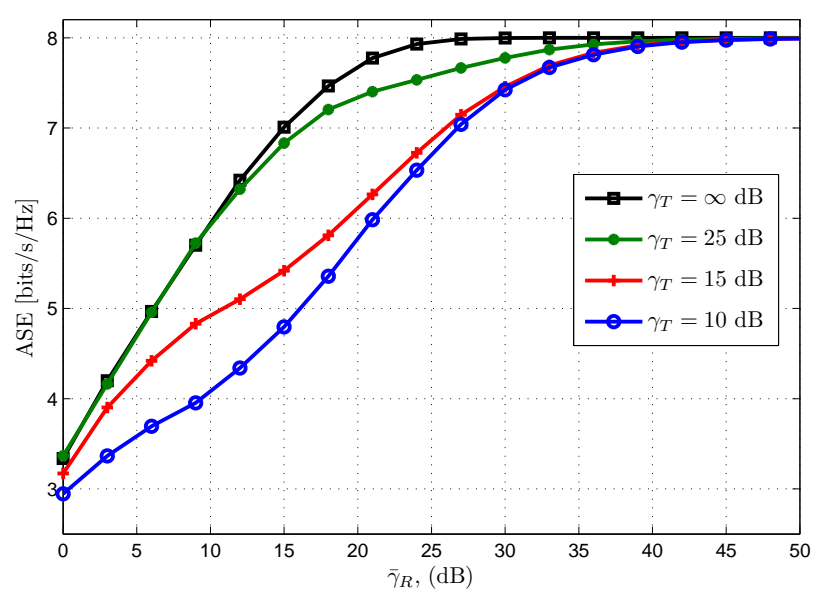

Fig. 1. Achievable average spectral efficiency for a target BER of $10^{-3}$ as a function of the average SNR at a distance $R$ from the BS for different values of $\gamma_{T}$.

Fig. 2 shows the average BER for the coded adaptive modulation scheme as a function of the average SNR at a distance $R$ from the base station for a target BER of $10^{-3}$ and different values of $\gamma_{T}$ when the number of users in the cell is $L=5$. It is clear that the BER performance is always less than the target value. The average BER performance improves as $\gamma_{T}$ increases, where the best performance is observed when $\gamma_{T} \rightarrow+\infty$.

In Fig. 3, the BER of the outdated channels estimates case is shown as a function of $\bar{\gamma}_{R}$ for different values of $\rho$ when $L=5$ and $\gamma_{T}=20 \mathrm{~dB}$. The figure shows the effect of the outdated channel estimates on the average BER performance. It is is seen that the imperfect channel estimates have noticeable effect on the average BER because for a certain average SNR, at a distance $R$ from the BS, range the BER is no longer less than the target $\mathrm{BER}_{0}$ and the BER performance is degraded more and more when $\rho$ decreases. 


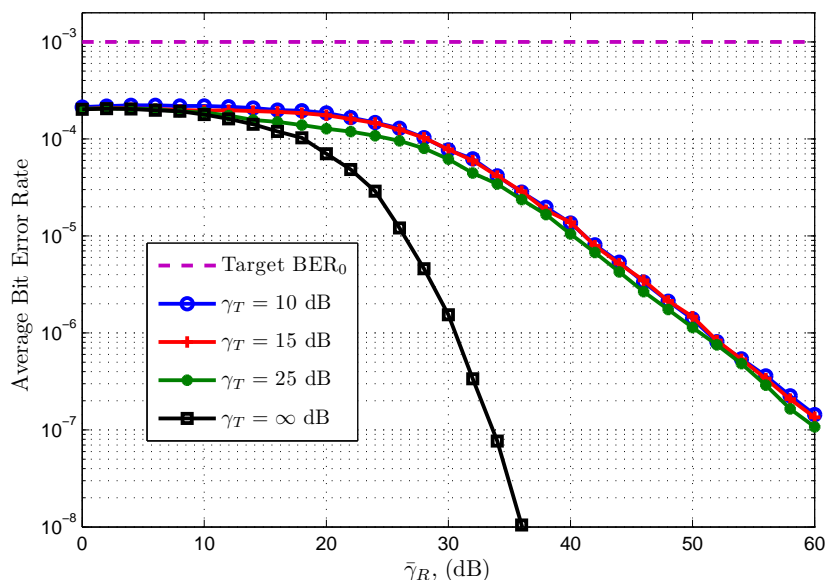

Fig. 2. Average BER for a target BER of $10^{-3}$ as a function of the average SNR at a distance $R$ from the BS for different values of $\gamma_{T}$.

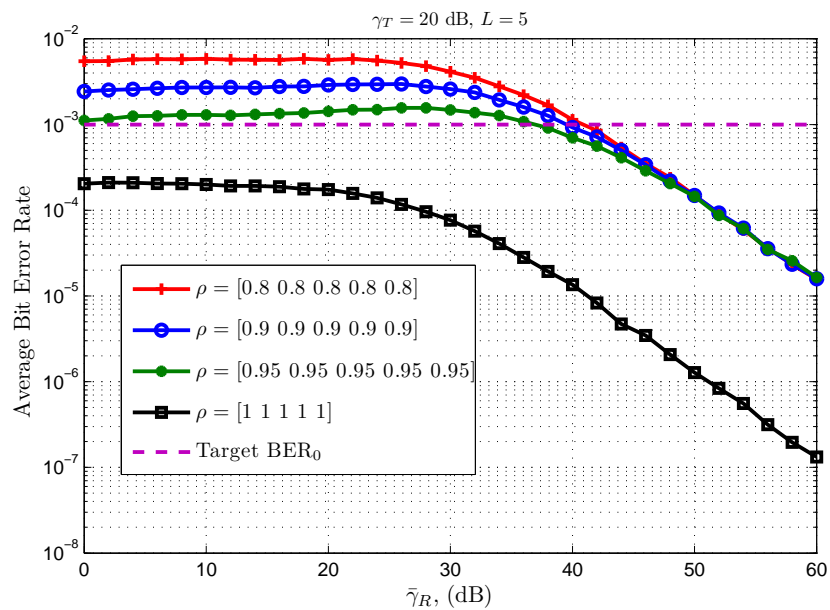

Fig. 3. Average BER in the outdated case for a target BER of $10^{-3}$ as a function of the average SNR at a distance $R$ from the BS for different values of $\rho$.

Fig. 4 depicts the AFL as a function of $\bar{\gamma}_{R}$ for different values of $\gamma_{T}$ when $L=5$. From Fig. 2 and Fig. 4, one can observe the trade-off between the ASE and AFL as a function of $\gamma_{T}$, where increase the ASE as $\gamma_{T}$ requires more users to be probed by the BS; hence the AFL is expected to increase. It is also clear that the AFL for the extreme case of $\gamma_{T} \rightarrow+\infty$ is always constant and equal to the number of users because full feedback from all users is needed to select the user with the best channel for each packet transmission.

\section{REFERENCES}

[1] R. Knopp and P. A. Humblet, Information capacity and power control in single-cell multiuser communications, in Proc. IEEE International Conference on Communications (ICC95), vol. 1, Seattle, Washington, pp. 331-335, Jun. 1995.

[2] P. Viswanath, D. N. C. Tse, and R. Laroia, "Opportunistic beamforming using dumb antennas," IEEE Transactions on Information Theory, vol.48, no.6, pp.1277-1294, Jun 2002

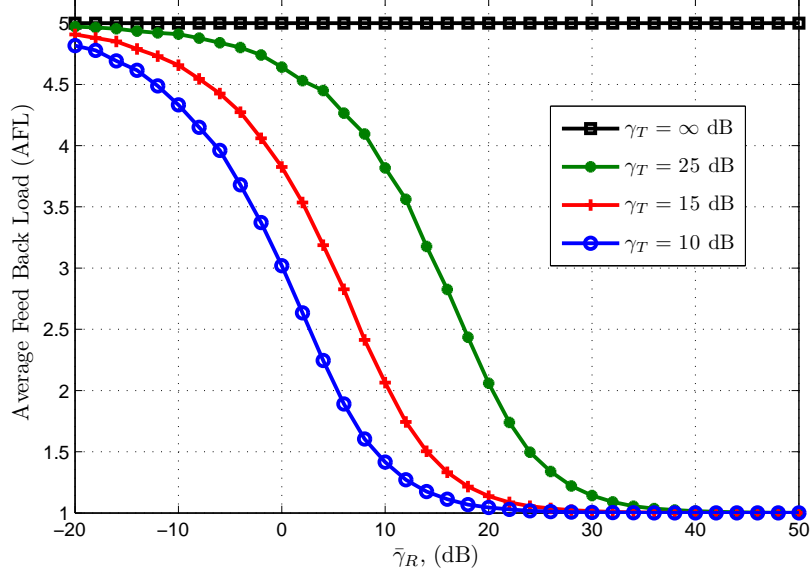

Fig. 4. Average feedback load as a function of the average SNR at a distance $R$ from the BS for different values of $\gamma_{T}$.

[3] H.-C. Yang and M.-S. Alouini. "Performance analysis of multi branch switched diversity systems," IEEE Transactions on Communications, vol. 51, no. 5, pp. 782-794, May 2003.

[4] H.-C. Yang and M.-S. Alouini, "Improving the performance of switched diversity with post-examining selection," IEEE Transactions on Wireless Communications, vol. 5, no. 1, pp. 67-71, January 2006.

[5] B. Holter, M.-S. Alouini, G. E. Oien, and H.-C. Yang, "Multiuser switched diversity transmission," in Proc. IEEE Vehicular Technology Conference (VTC2004-Fall), vol.3, Los Angeles, CA , pp. 2038-2043, Sept. 2004.

[6] M. K. Simon and M.-S. Alouini, Digital Communications over Generalized Fading Channels: A Unified Approach to Performance Analysis, New York, NY: John Wiley \& Sons, 2000.

[7] K. J. Hole, H. Holm, and G. E. Oien, "Adaptive multidimensional coded modulation over flat fading channels," IEEE Selected Areas in Communications, vol.18, no.7, pp. 1153-1158, Jul 2000

[8] M.-S.Alouini and A.J.Goldsmith, "Adaptive modulation over Nakagami fading channels," Kluwer Journal on Wireless Communications, vol. 13,pp. 119-143, 2000.

[9] I. S. Gradshteyn and I. M. Ryzhik, Tables of Integrals, Series, and Products, 7th ed., CA: Academic Press, 2007.

[10] L. Yang, M. Kang, and M.-S. Alouini, On the capacity-fairness tradeoff in multiuser diversity systems, IEEE Transactions on Vehicular Technology, vol. 56, no. 4 Part 1, pp. 1901-1907, 2007.

[11] A. H. Nuttall, "Some integrals involving the Q-function", Naval Underwater Systems Center, New London Lab., New London, Conn., Tech. Rep., 4297, Apr. 11, 1972.

[12] M. Schwartz, W. R. Bennett, and S. Stein, Communication Systems and Techniques. New York: McGraw-Hill, 1966.

[13] A. H. Nuttall, "Some integrals involving the $Q_{M}$ function," IEEE Trans. Inform. Theory, pp. 95-96, Jan. 1975. 